Volume 2, Issue 2, April-June 2017, Pages: 195, DOI: http://dx.doi.org/10.19082/ah195

\title{
GUIDELINES FOR A NATIONAL REGISTRY OF ACUTE CORONARY SYNDROME IN IRAN, BASED ON A COMPARATIVE STUDY IN SELECTED COUNTRIES
}

\author{
Hamidreza Tadayon ${ }^{1 *}$, Mahmoud Keyvanara ${ }^{2}$, Masoumeh Sadeghi ${ }^{3}$,Sakineh Saghaeiannejad Isfahani ${ }^{4}$
}

1: Ph.D. candidate of health Information Management, Kashan University of Medical Sciences, Kashan, Iran.

2: Associate professor of Medical Sociology, Social Determinants of Health Research Center, Isfahan University of Medical Sciences, Isfahan, Iran.

3: Professor of Cardiology, Cardiovascular Rehabilitation Research Center, Cardiovascular Research Center Institute, Isfahan University of Medical Sciences, Isfahan, Iran.

4: Lecturer, Health Information Technology Research Center, Isfahan university of Medical Sciences, Isfahan, Iran.

\section{Correspondence:}

Tel: +989395674435, E-mail: tadayon1363@gmail.com

\section{TYPE OF ARTICLE: CONFERENCE ABSTRACT}

\begin{abstract}
Introduction: Acute coronary syndrome (ACS) includes myocardial infarction (MI) with and without ST elevation and unstable angina (UA). This syndrome is a leading cause of morbidity and mortality in many countries. A registry system is a secondary collection of data regarding patients with special disease or procedures that can manage the disease data and decrease disease burden. Because a national registry of ACS does not exist in Iran, this research aims to present the guidelines for a national registry of ACS in Iran based on a comparative study in other countries.

Methods: This study was descriptive-comparative and applied research. Research community was ACS national registry of USA, Switzerland and Malaysia. The method of data gathering was to study all of the relevant documents about the research subject in other countries, and interview MI registry specialists in Isfahan cardiovascular research center. Analysis and identity of information was via comparative tables and subsequently, guidelines for ACS national registry in Iran were presented based on obtained findings.

Results: In all three countries, there was a national registry for ACS, and there is no national registry for ACS in Iran. ACS registry of selected countries is done in the following stages: data gathering, data processing and information distribution. This research reviewed the ACS national registry in selected countries, and after comparing obtained data, suggested guidelines for a national registry of ACS in Iran that include the seven following categories: structure of registry, purposes of registry, including and excluding criteria for a national registry in Iran, data gathering methods, data elements, data processing methods and information distribution methods.

Conclusion: For planning and implementing a national registry, gathering data is not enough but determining purposes is a basic stage. After this stage, determination of data elements and data analysis methods based on predetermined purposes, is next stage. Data must be completely defined in a data dictionary and should be properly analyzed.

KEYWORDS: Acute coronary syndrome, Myocardial infarction, Diseases registry, Comparative study.
\end{abstract}

\section{Abstracts of First National Congress of Medical Informatics, Mashhad, Iran, February 2017}

(C) 2017 The Authors. This is an open access article under the terms of the Creative Commons Attribution-NonCommercialNoDerivs License, which permits use and distribution in any medium, provided the original work is properly cited, the use is non-commercial and no modifications or adaptations are made. 